\title{
Eating attitudes: The extent and risks of disordered eating among amateur athletes from various sports in Gauteng, South Africa
}

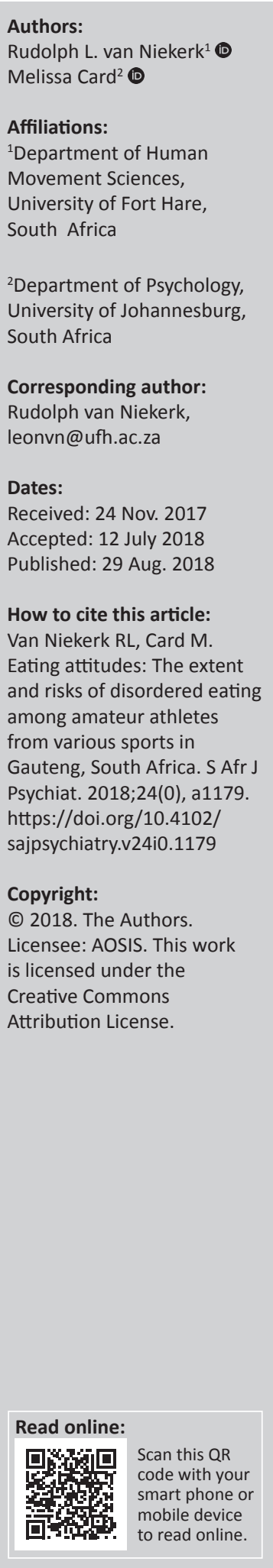

Background: Preoccupation around eating and disordered eating of professional athletes has extensively been discussed in the literature. However, the extent of disordered eating behaviours at the non-professional or amateur club level in South African sport has not received the same amount of attention.

Objectives: This study attempted to determine the extent of disordered eating behaviours among amateur athletes to identify the athletes at risk of developing an eating disorder. Group differences and predictive factors were explored to determine factors associated with disordered eating behaviours among amateur sporting athletes.

Methods: A purposive sample of athletes $(n=278)$ with a mean age of $27 \pm 11.30$ years, from various sports clubs in Central Gauteng (in and around Johannesburg), were asked to complete the Eating Attitudes Test-26 and Sport Competition Anxiety Test. Data analysis included descriptive statistics, independent samples $t$-tests, analysis of variance (ANOVA) and logistic regression.

Results: The results indicated that $14.7 \%$ of athletes were at risk of developing an eating disorder, while some engaged in excessive weight control behaviour which put them at risk. Gender and weight control strategies were important indicators associated with the risk of developing an eating disorder. The athletes' gender, level of participation and body mass index (BMI) were important predictors of the risk to develop an eating disorder.

Conclusion: Indicators of eating disorder risk among club-level amateur athletes are gender, binge eating, vomiting and using laxatives to control weight. These behaviours predominately found in female athletes seem to put them at a greater risk of developing an eating disorder.

\section{Introduction}

Eating attitudes and disordered eating behaviours among athletes is an area of research that needs much attention. This research attempts to address this problem by assessing disordered eating behaviour among athletes from various amateur sports. The general meaning of the term 'athlete' will be used in this article to refer to a sports participant in any of the sports included in the study. Within South Africa, there is no real focus on how athletes engage with eating and food in general. However, there is much focus on the prevalence of eating disorders in athletes, which seems to be similar to international research, and leads to the conceptualisation of the female athlete triad ${ }^{1}$ - that is, an interrelationship between disordered eating behaviours, amenorrhea and low bone density - which attests to the significant health risks for women participating in sport and places them at high risk of developing an eating disorder. One of the factors in this triad is disordered eating behaviours, which is seen as an inclination towards rapid weight loss and abnormal eating behaviours that are subclinical, as they do not meet the criteria for any diagnosable eating disorder. Over time, however, disordered eating behaviours can become so ingrained that they will develop into a diagnosable eating disorder among both female and male athletes. ${ }^{2,3}$ Eating disorder diagnosis in athletes is beyond the scope of this research; however, it aims to highlight how disordered eating behaviours exist among South African amateur athletes. Athletes are often under pressure to perform at best because of the nature of competition and it is not clear whether amateur athletes are engaging in similar disordered eating behaviour than some professional athletes that put them at risk for developing an eating disorder. By identifying the disordered eating behaviours of athletes, it is possible to identify the 'at risk' amateur athlete with the potential development of eating pathology. 
The idea of an 'at risk' of an eating disorder athlete is underestimated and needs more attention especially when viewed in light of the prevalence of eating disorders in sport. ${ }^{4}$ Research focussing on the prevalence of eating disorders among different athletes is largely inconclusive because of a number of mitigating factors, but it seems to be more prevalent among athlete than non-athlete populations, ${ }^{5}$ both during adolescence ${ }^{4}$ and adulthood. ${ }^{6}$ Prevalence rates also seem to be higher among female athletes than male athletes. ${ }^{4,6}$ Weight-sensitive sports such as endurance, weight category and aesthetic sports, jumping sports and dancing ${ }^{5}$ seem to be high-risk sports for the development of eating disorders or inappropriate behaviours when engaging with food. ${ }^{6}$ International studies have reported high variability in the prevalence rates of athletes at risk for eating disorders, from as low as $7 \%$ to as high as $62 \%{ }^{4,6,7}$ It is argued that the variability may be because of diagnostic and methodological issues. ${ }^{1}$ Similar inconsistent prevalence rates were found among South African athletes, from as low as $14 \%$ among runners ${ }^{8}$ to a substantially higher number of $54 \%$ among elite netball players $^{9}$ and $62.5 \%$ in student track and field athletes. ${ }^{10}$ Therefore, research on eating disorders in sport needs to be understood and interpreted with caution as results are often inconsistent. This necessitates more research to understand the disordered eating behaviours and its prevalence in amateur sports in South Africa. Similar research focussing on identifying the amateur athlete 'at risk' of developing disordered eating behaviours needs to be more prominent in order to assist in the prevention of the development of any eating pathology, including the role competitive anxiety ${ }^{11}$ plays in the context of amateur club-level sport.

\section{Method}

Previous international research ${ }^{1,2,4,5,6,7}$ focussing on eating disorders among sportsmen and sportswomen was conducted mostly on professional youth, student and adult athletes, often overlooking disordered eating behaviours among amateur athletes at club level. This study is exploratory in nature and attempts to determine the prevalence and risk factors of disordered eating behaviours and to identify amateur athletes 'at risk' of developing eating disorders at club level in South Africa. Group differences were determined for gender, type of sports and level of participation. The study also explored the relationship between variables and predictability of disordered eating behaviours among amateur athletes at club level in South Africa.

\section{Participants}

A purposive sample of 278 athletes with a mean age of $27 \pm$ 11.30 years from various amateur sports clubs representing 31 team and individual sports codes in urban areas in and around Johannesburg, Gauteng, were invited to participate in the study through a study information letter. Clubs that responded positively to the invitation were included in the study. The sample included both male and female athletes, who participated in provincial, national and international levels. Twenty field workers met with the athletes at their sport clubs and introduced about the purpose of the study. Athletes who volunteered to participate were asked to sign an informed consent form that ensured confidentiality, defined the parameters of the study and how the data will be used and published. Once the informed consent was signed, participants completed the questionnaires.

\section{Research instrument}

Athletes were asked to complete a biographical questionnaire, including their age, gender, weight, height, type of sports and level of participation. They were also requested to complete the Eating Attitudes Test-26 (EAT-26) ${ }^{12}$ and the Sport Competition Anxiety Test (SCAT) ${ }^{11}$

Eating Attitudes Test-26 is a widely used standardised measure of concerning symptoms that are characteristic of eating disorders. ${ }^{12}$ The EAT-26 does not provide a definitive diagnosis of a specific eating disorder, but rather serves as a screening measure to assess or identify individuals exhibiting symptoms of eating pathology that needs attention. ${ }^{12}$ It consists of 26 questions answered on a six-point Likert scale, ranging from 'always' to 'never'. Three subscales, dieting, bulimia and food preoccupation as well as oral control can be calculated from the 26 questions. The subscales can be defined as follows: ${ }^{12}$

1. Dieting is an indication of a pathological avoidance of fattening (high-calorie) foods and food preoccupation. Sport athletes with high scores on this scale are usually dissatisfied with their body shape and have a desire to be smaller.

2. Bulimia and food preoccupation is an indication of body image disturbance positively related to bulimia (binge eating and purging behaviour) and associated with a heavier body weight.

3. Oral control refers to self-control regarding food and social pressures to lose weight. The questionnaire also includes a section on behavioural assessment (four questions) to determine excessive weight control measures.

A score of 20 and above denotes a need to be referred to a professional to assess the presence of an eating disorder. High scores are not necessarily indicative of an eating disorder, but suggest concerns about an athlete's weight, shape and eating behaviours. Similarly, low scores are not necessarily indicative of the absence of such concerns, but should be read with caution because of denial of symptoms, which could occur in self-report instruments. ${ }^{12}$ The EAT-26 has high validity and reliability, with Cronbach's alpha scores between 0.83 and 0.90 for the three subscales and 0.90 for the scale as a whole. ${ }^{12}$ It must be noted however that the EAT-26 is a screening instrument with low predictive power ${ }^{13}$ and therefore results have to be interpreted with caution. High scores on the instrument must not be interpreted as 
an eating disorder without further screening or referral to a professional, as questions can be misinterpreted, specifically in cross-cultural contexts such as South Africa.

The SCAT ${ }^{11}$ consists of 15 questions of which 10 questions are used to calculate a competitive anxiety score. It is answered on a three-point Likert scale ranging from 'hardly ever' to 'sometimes' and 'often'. Norm scores were developed by the author ${ }^{11}$ and can be categorised to identify athletes with low (anxiety score $<16$ ), moderate (anxiety score $=17-22$ ) and high competitive anxiety (anxiety score $>23$ ). High reliability and validity is reported on the instrument with an internal consistency (Cronbach's alpha) of between 0.8 and $0.85 .{ }^{11}$

\section{Data analysis}

Descriptive statistics (means and standard deviations) and frequency tables were used to describe the participants. Statistical significance was set at $5 \%(p=0.05)$. The prevalence of disordered eating and risk factors was calculated as a percentage according to the norm scores provided with the EAT- $26^{12}$ and the internal consistency of the various subscales was determined with Cronbach's alpha. Group differences were determined using independent samples $t$-tests and analysis of variance (ANOVA) with Bonferonni post hoc tests. Finally, to determine which factors have a statistically significant contribution to the prediction of disordered eating, a logistic regression was performed.

\section{Ethical consideration}

This research project was approved by the University of Johannesburg's higher degrees and ethics committees before being conducted.

\section{Results}

The amateur athletes who participated in this study spent on average $8.71 \pm 5.79 \mathrm{~h}$ training per week. While most of them participated in club levels $(n=178,64.7 \%)$, the sample included amateur athletes participating in provincial $(n=37$, $13.5 \%)$ and national or international ( $n=60,21.8 \%)$ levels. The sample consisted of 161 (57.9\%) male and 117 (42.1\%) female participants, representing a fair distribution to determine group differences. Athletes from various team sports $(n=132,47.5 \%)$ and individual sports $(n=146,52.5 \%)$ were represented in the sample (see Table 1$)$. The team sports were mostly represented by Football (37.1\%), Rugby (16.7\%), Basketball (14.4\%) and Netball and Hockey (7.6\%). Athletics (36.3\%), Tennis and Boxing (9.6\%) and Squash (7.5\%) were represented mostly in individual sports.

The physical attributes and eating attitudes of the participating amateur athletes are presented in Table 2. The participants had a mean weight $(\mathrm{kg})$ of $68.87 \pm 14.20$ ranging between a minimum of $43 \mathrm{~kg}$ and a maximum of $118 \mathrm{~kg}$. The mean height $(\mathrm{m})$ of the participants was $1.69 \pm 0.14$, with a minimum of $1.20 \mathrm{~m}$ and a maximum of $2.30 \mathrm{~m}$. These measures were used to calculate the body mass index (BMI) of the participants. The mean BMI $(24.33 \pm 4.75)$ of the
TABLE 1: Frequencies of team and individual sports represented in the sample.

\begin{tabular}{|c|c|c|}
\hline$\overline{\text { Sport }}$ & Frequency $(n)$ & $\%$ \\
\hline \multicolumn{3}{|l|}{ Team sports } \\
\hline Football & 49 & 37.1 \\
\hline Rugby & 22 & 16.7 \\
\hline Basketball & 19 & 14.4 \\
\hline Netball & 10 & 7.6 \\
\hline Hockey & 10 & 7.6 \\
\hline Cricket & 5 & 3.8 \\
\hline Dancing & 5 & 3.8 \\
\hline Sailing & 4 & 3.0 \\
\hline Water Polo & 3 & 2.3 \\
\hline Touch Rugby & 2 & 1.5 \\
\hline Drum Majorette & 2 & 1.5 \\
\hline Softball & 1 & 0.8 \\
\hline Total & 132 & 47.5 \\
\hline \multicolumn{3}{|l|}{ Individual sports } \\
\hline Athletics & 53 & 36.3 \\
\hline Tennis & 14 & 9.6 \\
\hline Boxing & 14 & 9.6 \\
\hline Squash & 11 & 7.5 \\
\hline Gymnastics & 7 & 4.8 \\
\hline Judo & 7 & 4.8 \\
\hline Swimming & 6 & 4.1 \\
\hline Cycling & 6 & 4.1 \\
\hline Karate & 5 & 3.4 \\
\hline Golf & 4 & 2.7 \\
\hline Aerobics & 4 & 2.7 \\
\hline Wrestling & 3 & 2.1 \\
\hline Canoeing & 3 & 2.1 \\
\hline Ballet & 2 & 1.4 \\
\hline Weight lifting & 2 & 1.4 \\
\hline Kickboxing & 2 & 1.4 \\
\hline Rowing & 1 & 0.7 \\
\hline Lawn bowls & 1 & 0.7 \\
\hline Yoga & 1 & 0.7 \\
\hline Total & 146 & 52.5 \\
\hline
\end{tabular}

participants is within the normal category (presented in Table 2); however, upon further analysis, 5\% of the participants were found to be underweight, while $23.6 \%$ and $11.6 \%$ were found to be categorised as overweight and obese, respectively.

Both the SCAT and EAT-26 instruments yielded high reliability scores, with Cronbach's alpha at 0.829 and 0.841 , respectively, and thus contributed much towards the internal validity of the study. The results of the study could therefore be reported with confidence. The athletes' mean SCAT score for competitive anxiety $(20.45 \pm 4.13)$ fell within the moderate parameters of the test, which is appropriate. However, upon further analysis, one-third (33.1\%) of the athletes in the study seemed to have high competitive anxiety (see Table 2).

Table 2 also presents the EAT-26 scores of the participants. According to the norm scores, ${ }^{12}$ a score of 20 and more might be indicative of an athlete at risk of developing an eating disorder (subclinical eating disorder pathology). Although the mean score $(10.59 \pm 9.55)$ for these athletes is well below the cut-off score of 20, further analysis and categorisation of the scores showed that $14.7 \%$ of the amateur athletes in this sample seem to be at risk of developing an eating disorder. 
TABLE 2: Physical attributes, competitive anxiety (Sport Competition Anxiety Test scores) and eating attitudes (Eating Attitudes Test-26scores) of the sample.

\begin{tabular}{|c|c|c|c|c|c|c|}
\hline \multirow[t]{2}{*}{ Variable } & \multirow[t]{2}{*}{ Mean \pm s.d. } & \multirow[t]{2}{*}{ Minimum } & \multirow[t]{2}{*}{ Maximum } & \multirow[t]{2}{*}{ Categories } & \multicolumn{2}{|c|}{ Frequency } \\
\hline & & & & & $n$ & $\%$ \\
\hline \multirow[t]{4}{*}{ Body mass index } & $24.33 \pm 4.75$ & 12.70 & 45.14 & Underweight & 13 & 5.0 \\
\hline & & & & Normal & 155 & 59.8 \\
\hline & & & & Overweight & 61 & 23.6 \\
\hline & & & & Obese & 30 & 11.6 \\
\hline \multicolumn{7}{|l|}{ SCAT scores } \\
\hline \multirow[t]{3}{*}{ Competitive anxiety } & $20.45 \pm 4.13$ & 10 & 30 & Low & 35 & 12.6 \\
\hline & & & & Moderate & 151 & 54.3 \\
\hline & & & & High & 92 & 33.1 \\
\hline \multicolumn{7}{|l|}{ EAT-26 scores } \\
\hline \multirow[t]{2}{*}{ Eating attitude } & $10.59 \pm 9.55$ & 0 & 55 & No risk - ED & 233 & 85.3 \\
\hline & & & & Risk - ED & 40 & 14.7 \\
\hline Dieting & $8.49 \pm 6.02$ & 2 & 31 & Risk - AN & 20 & 7.30 \\
\hline Bulimia and food preoccupation & $2.14 \pm 2.44$ & 0 & 12 & Risk - BN & 6 & 2.19 \\
\hline
\end{tabular}

ED, eating disorder; AN, anorexia nervosa; BN, bulimia; OC, oral control; SCAT, Sport Competition Anxiety Test; EAT-26, Eating Attitudes Test-26; s.d., standard deviation.

TABLE 3: Disordered eating behaviour.

\begin{tabular}{lccc}
\hline Behaviour & Indicator & Frequency & $\mathbf{\%}$ \\
\hline Binge eating & No & 218 & 79.6 \\
& Yes & 56 & 20.4 \\
Vomiting to control weight & No & 240 & 87.6 \\
& Yes & 34 & 12.4 \\
Laxatives to control weight & No & 214 & 78.1 \\
& Yes & 60 & 21.9 \\
Exercise to control weight & No & 258 & 94.2 \\
& Yes & 16 & 5.8 \\
Lost 9 kg (20 Ib) or more in the & No & 231 & 84.3 \\
past 6 months & & & \\
& Yes & 43 & 15.7 \\
\hline
\end{tabular}

TABLE 4: Group differences for disordered eating among amateur athletes.

\begin{tabular}{llcc}
\hline Variable & Group & Mean \pm s.d. & Sign. $(p)$ \\
\hline Gender & Male & $8.87 \pm 8.38$ & 0.000 \\
& Female & $12.91 \pm 10.53$ & \\
Type of sports & Team sports & $10.30 \pm 10.20$ & 0.639 \\
& Individual sports & $10.85 \pm 8.98$ & \\
Level of participation & Club & $9.83 \pm 9.10$ & 0.275 \\
& Provincial & $12.14 \pm 9.21$ & \\
& National or international & $11.42 \pm 10.21$ & \\
Competitive anxiety & Low & $8.47 \pm 7.54$ & 0.282 \\
& Moderate & $10.52 \pm 9.78$ & \\
& High & $11.52 \pm 9.80$ & \\
\hline
\end{tabular}

s.d., standard deviation.

The disordered eating behaviour of the athletes (Table 3) provides an indication of the pathological attempts of amateur athletes to lose weight rapidly. Although most of the participants did not engage in such behaviours, $21.9 \%$ used laxatives, $20.4 \%$ engaged in binge eating and $12.4 \%$ purged to lose weight. In contrast, only $5.8 \%$ used exercise to lose weight. A total of $15.7 \%$ of the participants lost more than 9 $\mathrm{kg}(20 \mathrm{lb})$ in the past 6 months.

Gender, sport, level of participation and anxiety group differences for disordered eating are presented in Table 4. Although no group differences were found for the type of sports, the level of sport participation and the competitive anxiety of the athletes, a significant difference for disordered eating was found for the gender groups, with female athletes having significantly higher disordered eating scores $(M=12.91 \pm 10.53 ; t[272]=-3.53, p=0.000)$ than male athletes $(M=8.87 \pm 8.38)$ in the study. This implies that female athletes were much more 'at risk' to engage in problematic eating behaviours, putting them at greater risk of developing an eating disorder. The difference between the means yielded a small effect size (eta-squared $=0.044$ ). A chi-square test for independence indicated a significant association between gender and risk for an eating disorder $\left(\chi^{2}[1, n=273]=6.75\right.$, $p=0.009$, phi $=0.168)$, with only $9.6 \%$ of male athletes but $21.6 \%$ of female athletes at risk for developing an eating disorder.

A logistic regression analysis was performed to determine which factors play a significant role in the likelihood of amateur athletes developing an eating disorder. The logistic regression model consisted of nine independent variables (see Table 5): gender, type of sports, level of participation, $\mathrm{BMI}$, anxiety, binge eating, vomiting to control weight, use of laxatives to lose weight and exercise to control weight. The model was statistically significant with the omnibus test of model coefficients $\left(\chi^{2}[9, n=254]=25.32, p=0.003\right)$, while the Hosmer-Lemeshow goodness-of-fit test was not statistically significant $\left(\chi^{2}[8, n=254]=9.27, p=0.320\right)$. Thus, both tests indicated that the model was able to distinguish between participants who were at risk and who were not at risk of developing an eating disorder.

The model explained between 9.50\% (Cox and Snell's $R^{2}$ ) and $16.6 \%$ (Nagelkerke's $R^{2}$ ) of the variance in the risk to develop an eating disorder and classified $85 \%$ of the cases correctly. As presented in Table 5, three of the factors - gender ( $p=$ $0.007)$, level of participation $(p=0.019)$ and BMI $(p=0.025)-$ made a statistically significant contribution to the model. The strongest predictors of risk to develop an eating disorder were gender (odds ratio: 3.18), level of participation (odds ratio: 2.58), binge eating (odds ratio: 2.41) and vomiting to control weight (odds ratio: 2.16). This indicates that amateur athletes who are female, participate at provincial or international level, binge eat and purge to lose weight are two to three times more likely to be at risk of developing 
TABLE 5: Variables in the equation of the logistic regression.

\begin{tabular}{|c|c|c|c|c|c|c|c|c|}
\hline \multirow[t]{2}{*}{ Variables } & \multirow[t]{2}{*}{$B$} & \multirow[t]{2}{*}{ SE } & \multirow[t]{2}{*}{ Wald } & \multirow[t]{2}{*}{$d f$} & \multirow[t]{2}{*}{ Sig. } & \multirow[t]{2}{*}{$\operatorname{Exp}(B)$} & \multicolumn{2}{|c|}{$95 \% \mathrm{Cl}$ for $\operatorname{Exp}(B)$} \\
\hline & & & & & & & Lower & Upper \\
\hline Gender & 1.158 & 0.426 & 7.378 & 1 & 0.007 & 3.183 & 1.380 & 7.339 \\
\hline BMI & 0.084 & 0.037 & 4.994 & 1 & 0.025 & 1.087 & 1.010 & 1.170 \\
\hline Anxiety & -0.029 & 0.050 & 0.340 & 1 & 0.560 & 0.971 & 0.880 & 1.071 \\
\hline Type of sports & -0.164 & 0.400 & 0.168 & 1 & 0.682 & 0.849 & 0.388 & 1.860 \\
\hline Level of participation & 0.948 & 0.402 & 5.548 & 1 & 0.019 & 2.581 & 1.173 & 5.680 \\
\hline Vomiting & 0.771 & 0.570 & 1.830 & 1 & 0.176 & 2.162 & 0.707 & 6.607 \\
\hline Laxatives & 0.018 & 0.484 & 0.001 & 1 & 0.971 & 1.018 & 0.394 & 2.629 \\
\hline Exercise & 0.066 & 0.741 & 0.008 & 1 & 0.929 & 1.069 & 0.250 & 4.563 \\
\hline Constant & -4.476 & 1.494 & 8.971 & 1 & 0.003 & 0.011 & - & - \\
\hline
\end{tabular}

Note: Variable(s) entered on step 1: All.

$\mathrm{BMI}$, body mass index; $\mathrm{SE}$, standard error; $\mathrm{Cl}$, confidence interval.

an eating disorder, when controlling for all the other factors in the model.

\section{Discussion}

The results presented above reflect those of a purposive sample of amateur athletes from various sports clubs in Johannesburg, Gauteng, and as such cannot be generalised to other contexts. The results, however, indicate that there could be concerns for athletes being at risk of developing eating disorders, as disordered eating behaviour is prevalent in this group of athletes. The prevalence rate of $14.7 \%$ for amateur athletes at risk of developing an eating disorder participating at club level is consistent with the results found in other international studies. ${ }^{4,6,7}$ However, the results of those studies determined the prevalence rate of professional athletes. This suggests that the risk of developing an eating disorder is just as prevalent among club sport athletes as for professional sport athletes. Although the type of sport, the level of participation and anxiety did not indicate differences in the risk to develop an eating disorder, the results indicated that female athletes are more at risk of engaging in disordered eating behaviours, leaving them vulnerable to eating disorders. This result is consistent with previously conducted international studies in sport. ${ }^{1,2,3,14}$ This is an indication that female athletes at amateur level are just as vulnerable to eating disorders as their international counterparts, and sports managers and coaches should thus be aware and sensitive not to aggravate the issue and, as a preventative strategy, should refer athletes to psychiatrists or clinical psychologists when disordered eating behaviour is observed. Female athletes are perhaps more prone to the development of eating disorders because of body dissatisfaction and as a result pre-occupied with food, body weight and appearance. ${ }^{14}$ The results of this study show that gender, level of participation and weight control strategies are important indicators associated with the risk of developing an eating disorder. Factors influencing this need could arise from social media exerting the ideal symbolisation of health and fitness, and female sport athletes comparing themselves to their fellow sport athletes. However, female athletes reported that they are more likely than their male counterparts to use exercise to control their weight, mood, health and body tone. ${ }^{15}$
Furthermore, the results suggest that for the most part, the eating attitudes of the amateur athletes who participated in the study were within normal limits. However, more than one in five athletes indicated that they engaged in binge eating behaviour along with using laxatives to control weight. Amateur athletes in this study were less likely to use exercise as a means to lose weight and would rather engage in purging or using laxatives to do so. This could be attributed to gender, as women generally suffer from disturbed eating behaviours. ${ }^{16}$ The results of the study also indicated that there is a significant gender difference regarding eating disorder risk, thus making it more likely that female athletes would express that they have engaged in binge eating and the use of laxatives to control weight. ${ }^{17}$ Researchers have found that female athletes are more likely to report and engage in weight loss behaviours or disordered eating such as binge eating, rigorous dieting, fasting, vomiting and the use of laxatives and diuretics compared to men. ${ }^{16,17}$

It must be noted that weight loss among athletes is not necessarily related to an eating disorder. However, one can assume from the results of the study that a fair amount of amateur athletes who engage in at least one of the weight control measures have at least one risky eating behaviour. This could possibly leave them vulnerable to a perpetual cycle of not knowing the correct nutritional information needed, thus leading to disordered eating behaviours. These research findings coincide with other research, ${ }^{18}$ reporting that a pattern of eating habits of dieting during an athletic career may predispose athletes to later and more severe eating disorders within both genders. It seems that gender, level of participation and BMI of the athletes are important contributors to the prediction of the risk of developing an eating disorder. A model that included nine factors and was able to correctly classify more than $85 \%$ of cases can be utilised by coaches and medical or health professionals involved to be more aware of athletes who are at risk of an eating disorder. Officials, such as coaches who are aware of athletes who are overweight and engaged in disordered eating behaviour, should consult medical or health professionals available to the athletes to address the risk sensitively and appropriately. 
Limitations of the study are primarily methodological in nature. The sample was purposive and only selected from a local population in Johannesburg. The results are thus not generalisable. Only athletes from clubs who responded to the researchers' invitation were included; this could contribute to the possible underestimation of the results because of non-participation. It is suggested that future research strategies should include random selection of participants. Furthermore, although the sample included a variety of 31 different sports codes, it is relatively small and could be expanded in future research to include a larger population of athletes representative of other provinces in South Africa, as well as athletes from rural populations. The sample is also non-clinical and heterogeneous as the purpose of the study was exploratory. Future research should be conducted to include clinical homogeneous populations in sports that could be more advantageous to psychiatry. Another limitation of this study is that no pilot study was conducted to attend to possible problems with item interpretation. Although the SCAT was validated for the South African context, ${ }^{19}$ it is not so for the EAT-26, as its predictive power is low and results would need to be confirmed by further screening by professionals. Although both questionnaires had high internal consistency, it is suggested that future research should be conducted on validating the EAT-26 for the South African context.

\section{Conclusion}

Indicators of eating disorder risk among club-level athletes are gender, BMI, level of participation, binge eating, vomiting and using laxatives to control weight. These behaviours predominately found in female amateur athletes seem to put them at a greater risk of developing an eating disorder. All of the disturbed eating patterns that female amateur sport athletes seem to engage in are consistent with people who may have subclinical symptoms of anorexia nervosa, bulimia nervosa, binge eating disorder or eating disorders not otherwise specified.

\section{Acknowledgements Competing interests}

The authors declare that they have no financial or personal relationships which may have inappropriately influenced them in writing this article.

\section{Authors' contributions}

R.L.v.N. was the main researcher and M.C. was the collaborative researcher. R.L.v.N. conceptulised the study, performed data analysis and edited the article. M.C. did literature survey and wrote the first draft of the article. Both authors read and approved the final manuscript.

\section{References}

1. Nattiv A, Loucks AB, Manore MM, et al. American college of sports medicine position stand. The female athlete triad. Med Sci Sports Exerc. 2007:39(10):18671882. https://doi.org/10.1249/mss.0b013e318149f111

2. Filaire $E$, Truevelot $P$, Toumi $H$. Relationship between eating-behaviour disorders and psychological parameters in male first-year physical education students. Int J Sport Nutr Exerc Metab. 2012;22(5):383-391. https://doi.org/10.1123/ijsnem.22.5.383

3. McArdle WD, Katch FI, Katch VL. Sports and exercise nutrition. 3rd ed. Baltimore, MD: Lippincott. Williams \& Wilkins; 2009.

4. Martinsen $M$, Sundgot-Borgen J. Higher prevalence of eating disorders among adolescent elite athletes than controls. Med Sci Sports Exerc. 2013;45(6): 1188-1197. https://doi.org/10.1249/MSS.0b013e318281a939

5. Smolak L, Murnen SK, Ruble AE. Female athletes and eating problems: A metaanalysis. Int J Eat Disord. 2000;27:371-380. https://doi.org/10.1002/(SICI)1098108X(200005)27:4\%3C371::AID-EAT1\%3E3.0.CO;2-Y

6. Sundgot-Borgen J, Torstveit MK. Prevalence of eating disorders in elite athletes is higher than in the general population. Clin J Sports Med. 2004;14(1):25-32. https://doi.org/10.1097/00042752-200401000-00005

7. Theodorakou K, Donti O. Prevalence of eating disorders and psychologica parameters in elite female gymnasts: The relation to body image and body mass index. Athlitiki Psychologia. 2013;24:1-4.

8. Weight LM, Noakes TD. Is running an analog of anorexia?: A survey of the incidence of eating disorders in female distance runners. Med Sci Sports Exec. 1987;19(3):213-217. https://doi.org/10.1249/00005768-198706000-00005

9. Havemann L, De Lange Z, Pieterse K, Wright HH. Disordered eating and menstrual patterns in female university netball players. S Afr J Sport Med. 2011;23(3):68-72. https://doi.org/10.7196/sajsm.260

10. Robbeson JG, Havemann-Nel L, Wright, HH. The female athlete triad in student track and field athletes. S Afr J Clin Nutr. 2013;26(2):19-24. https://doi.org/10. 1080/16070658.2013.11734446

11. Martens R. Sport competition anxiety test. Champaign, IL: Human Kinetics; 1982.

12. Garner DM, Olmsted MP, Bohr $Y$, et al. The eating attitudes test: Psychometric features and clinical correlates. Psychol Med. 1982;12(4):871-878. https://doi. org/10.1017/S0033291700049163

13. Szabo CP, Allwood CW. Application of the Eating Attitudes Test (EAT-26) in a rural, Zulu speaking, adolescent population in South Africa. World Psychiatr. 2004;3(3):169-171.

14. Sundgot Borgen J, Torstveit MK. Aspects of disordered eating continuum in elite high intensity sports. Scand J Med Sci Sports 2010;20(s2):112-121. https://doi org/10.1111/j.1600-0838.2010.01190.x

15. Furnham A, Badmin N, Sneade I. Body image dissatisfaction: Gender differences in eating attitudes, self-esteem, and reasons for exercise. J Psychol. 2002, 136(6):581-596. https://doi.org/10.1080/00223980209604820

16. Kiefer I, Rathmanner T, Kunze M. Eating and dieting differences in men and women. J Men's Health Gend. 2005;2(2):194-201. https://doi.org/10.1016/j. jmhg.2005.04.010

17. Weinberg RS, Gould D. Foundations of sport and exercise psychology. 5th ed. Champaign, IL: Human Kinetics; 2011.

18. Stirling A, Kerr G. Perceived vulnerabilities of female athletes to the development of disordered eating behaviours. Eur J Sport Sci. 2012;12(3):262-273. https://doi. org/10.1080/17461391.2011.586437

19. Potgieter J. Norms for the Sport Competition Anxiety Test (SCAT). S Afr J Res Sport Phys Educ, Recreation. 2009;31(1):69-79. https://doi.org/10.4314/sajrs.v31i1.43793 